\section{Über konvexe Mengen in linearen normierten Räumen}

von

S. MAZUR (Lwów).

Im folgenden teile ich einige Resultate über konvexe Mengen in linearen, normierten Räumen mit. Zuerst zeige ich, daß wenn eine lineare Mannigfaltigkeit $R$ keinen inneren Punkt eines konvexen Körpers $K$ enthält, es eine Hyperebene $H$ gibt, so daß $R \subset H$ und $K$ auf einer Seite von $H$ liegt. Daraus folgt, daß durch jeden Punkt einer konvexen Hyperfläche $F$ wenigstens eine Stützhyperebene von $F$ geht. Dieser Satz wurde schon von $\mathrm{H}$. MinkowsKr im Falle eines euklidischen Raumes bewiesen und in letzter Zeit von Herrn G. Ascoli auf lineare, normierte und separable Räume verallgemeinert ${ }^{1}$ ). Ich gebe dann notwendige und hinreichende Bedingungen dafür, da $\mathrm{B}$ in einem Punkte einer konvexen Hyperfläche $F$ eine Tangentialhyperebene von $F$ existiert und zeige weiter, daß im Falle eines separablen Raumes vom Typus $(B)$ die Menge $Z$ der Punkte einer konvexen Hyperfläche $F$ in denen die Tangentialhyperebene von $F$ existiert, eine in $F$ dichte $G_{\delta}$-Menge bildet; $F-Z$ ist also in $F$ von erster Kategorie. Die oben formulierten Sätze bilden den Inhalt von $\S 1$. Im $\S 2$ beweise ich vor allem, daß jede konvexe abgeschlossene Menge schon schwachabgeschlossen ist; anders gefaßt lautet dieser Satz: wenn die Punktfolge $\left\{x_{n}\right\}$ gegen den Punkt $x_{0}$ schwach konvergiert, so gibt es zu jeder Zahl $\varepsilon>0$, Zahlen $c_{n} \geqslant 0(n=1,2, \ldots$ m) mit der Summe 1, so daß $\left|\sum_{n=1}^{m} c_{n} x_{n}-x_{0}\right|<\varepsilon$. Dieser Satz

1) H. Minkowski, Theorie der konvexen Körper, insbesondero Begründung ihres Oberflächenbegriffs, Ges. Abh. 2 (1911) p. 131-229, insb. p. 139; G. Ascoli, Sugli spazi lineari metrici e le loro varietà lineari, Ann. di Mat. 10 (1932) p. $33-81$ und p. $203-232$, insb. p. 205. wurde im Falle der Räume $\left(L^{p}\right),\left(l^{p}\right)(p>1)$ von den Herren S. Banach und S. SaKs, im Falle des Raumes $(C)$ von den Herren D. C. Gillessie und W. A. Hurwitz, sowie unabhängig von Herrn Z. ZaLCWASSER bewiesen ${ }^{2}$ ); aus dem letztgenanten Resultate kann übrigens - wie ich mit Herrn BANACH bemerkt habe - der obige Satz in voller Allgemeinheit gefolgert werden, da jeder lineare, normierte und separable Raum mit einer linearen Menge des Raumes $(C)$ isometrisch ist $\left.{ }^{3}\right)$. Ist die Punktfolge $\left\{x_{n}\right\}$ gegen $x_{0}$ schwachkonvergent, so enthält also insbesondere jeder konvexe Körper mit unendlich vielen Gliedern der Folge $\left\{x_{n}\right\}$ auch $x_{0}$; es wird gezeigt, daß die Umkehrung davon auch richtig ist. Setzt man dabei voraus, daß die Folge $\left\{x_{n}\right\}$ beschränkt ist, so genügt es nur beschränkte konvexe Körper mit Mittelpunkt in Betracht zu ziehen. Ich füge endlich einige Bemerkungen an über die verallgemeinerten Minkowskischen Räume ${ }^{4}$ ).

\section{$\S 1$.}

Sei $E$ ein linearer und normierter Raum. Ist $x_{0} \in E$, so erklären wir die Abbildung $U(x)=x+x_{0}$ des Raumes $E$ auf sich selbst als Translation (um $x_{0}$ ). Mengen, die man mittels Translation aus linearen Mengen erhält, nennen wir lineare Mannigfaltigkeiten. Eine lineare abgeschlossene Mannigfaltigkeit $H \neq E$ bezeichnen wir als eine Hyperebene, wenn es keine lineare (abgeschlossene) Mannigfaltigkeit $H^{*} \neq E$ gibt, die $H$ als echte Teilmenge enthält. Man kann leicht beweisen, daß wenn $F(x)$ ein lineares Funktional $\equiv 0$ und $c$ eine $\mathrm{Zahl}$ ist, so bildet die Menge der Punkte $x$, für die $F(x)-c=0$ stattfindet, eine Hyperebene; umgekehrt gibt es für jede gegebene Hyperebene $H$ ein lineares Funktional $F(x)$ sowie eine Zahl $c$ derart, daß die Menge der Punkte $x$, für die $F(x)-c=0$ gilt, mit $H$ identisch ist. Diese Sätze kann man mit der Bemerkung vervollständigen: sind $F_{1}(x)$,

2) S. Banach et S. Saks, Sur la convergence forte dans les champs $L^{p}$. Stud. Math. 2 (1930) p. 51-57; D. C. Gillespie and W. A. Hurwitz, On sequences of continuous functions having continuous limits, Trans. Amer. Math. Soc. 32 (1930) p. 527-543; Z. Zalcwas ser, Sur une propriété du champ des fonctions continues, Stud. Math. 2 (1930) p. 63-67.

3) S. Banach, Théorie des opérations linéaires, Warszawa, 1932, p. 185.

4) Was die Bezeichnungen und Terminologie betrifft, s. das unter ${ }^{3}$ ) zitierte Buch; vgl. insb. p. 246. Die Sätze 1, 3 und 4 wurden von mir der Polnischen Mathematischen Gesellschaft (Abteilung Lwów) in der Sitzung vom 22. 5. 1932 mitgeteilt. 
$F_{2}(x)$ gegebene lineare Funktionale $\equiv 0$ und $c_{1}, c_{2}$ Zahlen, so bestimmen die Gleichungen $F_{1}(x)-c_{1}=0, F_{2}(x)-c_{2}=0$ dieselbe Hyperebene dann und nur dann, wenn es eine Zahl $a \neq 0$ gibt, so daß identisch $F_{2}(x)=a F_{1}(x)$ und $c_{2}=a c_{1}$ ist. Wir sagen, daß die Menge $A$ auf einer Seite der Hyperebene $H$ liegt, wenn für beliebige $x, y \in A-H, x \neq y$, die Strecke $x y$ keinen Punkt der Menge $H$ enthält. Es ist leicht einzusehen, daß damit die Menge $A$ auf einer Seite der durch die Gleichung $F(x)-c=0$, bestimmten Hyperebene liege, wo $F(x)$ ein lineares Funktional $\equiv \equiv 0$ und $c$ eine Zahl bedeutet, notwendig und hinreichend ist, daß das Funktional $F(x)-c$ in $A$ keine Zeichenänderung erleide.

Eine Menge soll ein konvexer Körper heißen, wenn sie abgeschlossen und konvex ist, sowie innere Punkte besitzt; den Rand eines konvexen Körpers bezeichnen wir als eine konvexe Hyper fläche. Der konvexe Körper $K$ enthalte 0 als einen inneren Punkt; wir bezeichnen dann für jedes $x$ mit $K(x)$ die untere Grenze der Menge der Zahlen $h>0$ von der Eigenschaft, daß $\frac{1}{h} x \in K$. Das auf diese Weise im Raume $E$ erklärte Funktional $K(x)$ nennen wir das Minkowskische Funktional von $K^{5}$ ).

1. Es ist $K(x)<1$, wenn $x$ ein innerer, $K(x)=1$, wenn $x$ ein Rand-, $K(x)>1$, wenn $x$ ein äußerer Punkt von $K$ ist.

2. Sind $x, y \in E$ und $t$ eine $Z a h l>0$, so ist: $1^{\circ} K(x) \geqslant 0$; $2^{\circ} K(x+y) \leqslant K(x)+K(y) ; 3^{\circ} K(t x)=t K(x) ; 4^{\circ} K(x) \leqslant M|x|$ ( $M$ eine Konstante).

Aus diesen Bemerkungen folgt u. a., daß wenn $x, y$ zwei verschiedene Punkte eines konvexen Körpers $K$ sind und dabei mindestens einer von ihnen dem Inneren von $K$ angehört, so ist jeder von $x$ und $y$ verschiedene Punkt der Strecke $x y$ ein innerer Punkt von $K$. Daraus folgern wir, daß das Innere eines konvexen Körpers konvex ist und daß jeder konvexe Körper die abgeschlossene Hülle seines Inneren bildet; offenbar ist die abgeschlossene Hülle einer offenen konvexen Menge ein konvexer Körper.

3. Ist $K(x)$ ein in $E$ erklärtes Funktional mit den in der Bemerkung 2 angegebenen Eigenschoften $1^{\circ}-4^{\circ}$, so stellt die

5) Die Bemerkungen 1, 2, 3 und 5 sind für den. Fall eines euklidischen Raumes in der unter ${ }^{1}$ ) zitierten Arbeit von Minkowski, p. 132-135, enthalten die Bemerkungen 1-4 im Falle der allgemeinen linearen normierten Rüume wurden von Herrn Ascoli in der unter ${ }^{1}$ ) zitierten Arbeit, p. 48-50, bewiesen.
Menge der Punkte $x$, für die $K(x) \leqslant 1$ ist, einen konvexen Körper $K$ mit 0 als inneren Punkt wor; $K(x)$ ist das Minkozwskische Funktional von $K$.

Setzt man wieder voraus, daß $K$ ein konvexer, 0 im Inneren enthaltender Körper ist, so kann man folgendes leicht beweisen: 4. Damit $K$ beschränkt sei, ist notwendig und hinreichend, daß $K(x) \geqslant m|x|$ ( $m$ eine positive Konstante) für $x \in E$ sei.

Ist $H$ eine Hyperebene und $K$ ein konvexer Körper, so liegt offenbar $K$ dann und nur dann auf einer Seite von $H$, wenn $H$ keinen inneren Punkt von $K$ enthält. Wir sagen, daß die Hyperebene $H$ eine Stützhyperebene von $K$ (wie auch von seinem Rande $F$ ) ist, wenn $K$ auf einer Seite von $H$ liegt und dabei die Entfernung der Mengen $K, H$ gleich Null ist. Wenn 0 dem Inneren von $K$ angehört, so gilt nun die folgende Bemerkung:

5. Damit $K$ auf einer Seite der durch die Gleichung $F(x)$ $-1=0$, wo $F(x)$ ein lineares Funktional $\equiv 0$ bezeichnet, bestimmten Hyperebene $H$ liege, ist notwendig und hinreichend, daß $F(x)$ $\leqslant K(x)$ für $x \in E$ sei. - Liegt $K$ auf einer Seite von $H$, so ist wegen $F(0)-1<0$ schon stets $F(x)-1 \leqslant 0$ für $x \in K$. Ist $K(x) \neq 0$, so gilt, nach $2, K\left(\frac{1}{K(x)} x\right)=1$, woraus, nach $1, \frac{1}{K(x)} x \in K$ folgt; somit $F\left(\frac{1}{K(x)} x\right)-1 \leqslant 0$ d. h. $F(x) \leqslant K(x)$. Dies gilt noch in dem Falle, wenn $K(x)=0$ ist; zufolge 2 ist dann nämlich $K(n x)=0$ $(n=1,2, \ldots)$, also nach 1 stets $n x \in K$ und daher $F(n x)-1 \leqslant 0$, was $F(x) \leqslant 0$ impliziert. Umgekehrt: aus $F(x) \leqslant K(x)$ für $x \in E$ folgt $F(x)-1 \leqslant 0$ für $x \in K$, da auf Grund von $1 K(x) \leqslant 1$ für $x \in K$ ist.

Satz 1. Enthält die lineare Mannigfaltigkeit $R$ keinen inneren Punkt des konvexen Körpers $K$, so gibt es eine Hyperebene $H$ derart, daß $R \subset H$ und $K$ auf einer Seite von $H$ liegt.

Beweis. Man kann voraussetzen, das $R$ abgeschlossen ist und $K$ den Punkt 0 im Inneren enthält. Sei $r_{0} \in R$ und $S$ die Menge der Elemente der Form $r+t r_{0}$, wo $r \in R, t$ eine Zahl ist. Ersichtlich ist $S$ eine lineare Menge; sie kann als linearer normierter Raum aufgefaßt werden. $K S$ ist in $S$ ein konvexer Körper; wir zeigen jetzt, daß $R$ in diesem Raume eine Hyperebene bildet. Die lineare Mannigfaltigkeit $R$ ist offenbar in $S$ abge- 
schlossen und $\neq S$. Sei $R^{*}$ eine lineare Mannigfaltigkeit, so daß $R \subset R^{*} \subset S$ und $r_{1}+t_{1} r_{0} \in R^{*}$, wo $r_{1} \in R, t_{1}$ eine Zahl $\neq 0$ ist. Bei gegebenen $r \in R$ sowie einer Zahl $t$, setzen wir $r_{2}=\frac{t(1-\alpha)}{t-a t_{1}} r_{0}$ $+\frac{a t}{t-a t_{1}} r_{1}-\frac{a t_{1}}{t-a t_{1}} r, r_{3}=(1-a) r_{0}+a\left(r_{1}+t_{1} r_{0}\right)$, wo $a$ eine $\mathrm{Zahl} \neq 0$ und $\neq \frac{t}{t_{1}}$ ist. Aus $r_{0}, r_{1}, r \in R$ und $\frac{t(1-a)}{t-a t_{1}}+\frac{a t}{t-a t_{1}}$ $-\frac{a t_{1}}{t-a t_{1}}=1$ folgt $r_{2} \in R$, mithin auch $r_{2} \in R^{*}$; analog wegen $r_{0}, r_{1}+t_{1} r_{0} \in R^{*}, \quad(1-a)+a=1$, ist $r_{3} \in R^{*}$. Da $r_{2}, r_{3} \in R^{*}$ und $\left(1-\frac{t}{a t_{1}}\right)+\frac{t}{a t_{1}}=1$, so ergibt die Gleichheit $r+t r_{0}=\left(1-\frac{t}{a t_{1}}\right) r_{2}$ $+\frac{t}{a t_{1}} r_{3}$ sofort $r+t r_{0} \in R^{*}$. Auf diese Weise ist bewiesen, dafs $R^{*}=S ; R$ bildet also tatsächlich in $S$ eine Hyperebene. Es gibt daher in $S$ ein lineares Funktional $f(x)$ derart, daß die Menge der $x \in S$, für die $f(x)-1=0$ gilt, mit $R$ übereinstimmt. Da $K S$ offenbar auf einer Seite von $R$ liegt, ist nach $5 f(x) \leqslant \dot{K}(x)$ für $x \in S$. Wegen 2 ist $K(x+y) \leqslant K(x)+K(y), K(t x)=t K(x)$ für $x, y \in E$ und Zahlen $t>0$; andererseits ist das in $S$ erklärte Funktional $f(x)$ additiv, homogen und dabei $f(x) \leqslant K(x)$ für $x \in K$. Folglich gibt es in $E$ ein additives und homogenes Funktional $F(x)$ derart, daß stets $F(x) \leqslant K(x)$ und $F(x)=f(x)$ für $\left.x \in S^{0}\right)$; da außerdem, zufolge $2, K(x) \leqslant M|x|$ (M eine Konstante) und somit auch $F(x) \leqslant M|x|$ für $x \in E$ ist, so ist das Funktional $F(x)$ stetig. Die durch die Gleichung $F(x)-1=0$ bestimmte $\cdot$ Hyperebene $H$ besitzt die verlangten Eigenschaften; es ist $R C H$ und nach 5 liegt $K$ auf einer Seite von $H$.

Aus diesem Satze folgt insbesondere, daß durch jeden Punkt einer konvexen Hyperfläche $F$ wenigstens eine Stützhyperebene von $F$ geht. Betrachten wir z. B. den konvexen Körper $K_{r}$, welchen die Kugel $|x| \leqslant r(r$ eine Zahl $>0)$ bildet; das Minkowskische Funktional von $K_{r}$ ist $\frac{1}{r}|x|$. Nach 5 stellt die Gleichung $F(x)-r=0$, wo $F(x)$ ein lineares Funktional $\equiv \equiv 0$ bezeichnet, dann und nur dann eine durch den Randpunkt $x_{0}$ von $K_{r}$ gehende $\left.{ }^{8}\right)$ S. das unter ${ }^{3}$ ) zitierte Buch, p. 27.
Stützhyperebene von $K_{r}$ dar; wenn $\|F\|=1$ und $F\left(x_{0}\right)=\left|x_{0}\right|$ ist; der Satz 1 impliziert also u. a. die Existenz von linearen Funktionalen mit den obigen Eigenschaften.

6. Sei $p(x)$ ein im linearen Raume $E$ erklärtes Funktionaı derart, daß für $x, y \in E$ und Zahlen $t>0: 1^{\circ} \varphi(x+y) \leqslant \varphi(x)$ $+\varphi(y), 2^{\circ} \varphi(t x)=t \varphi(x)$ sei; sei ferner $x_{0} \in E$. Dann existiert der endliche $\lim _{h \rightarrow+0} \frac{1}{h}\left[\varphi\left(x_{0}+h x\right)-\varphi\left(x_{0}\right)\right]$ für $x \in E$, und $\Phi(x)=\lim _{h \rightarrow+0} \frac{1}{h}$ $\left[\varphi\left(x_{0}+h x\right)-\varphi\left(x_{0}\right)\right]$ gesetzt, ist für $x, y \in E$ und Zahlen $t>0$ : $\left.\overline{1^{\circ}} \Phi(x+y) \leqslant \Phi(x)+\Phi(y) ; \overline{2^{\circ}} \Phi(t x)=t \Phi(x) ; \overline{3^{\circ}} \Phi(x) \leqslant \varphi(x)^{\eta}\right)$. Setzen wir $R(x, h)=\frac{1}{h}\left[\varphi\left(x_{0}+h x\right)-\varphi\left(x_{0}\right)\right]$ für $x \in E$ und positive Zahlen $h$. Sind $x \in E$ und $h_{1}, h_{2}$ Zahlen derart, daß $0<h_{1}<h_{2}$, so ist $h_{2} p\left(x_{0}+h_{1} x\right)=p\left(h_{1}\left(x_{0}+h_{2} x\right)+\left(h_{2}-h_{1}\right) x_{0}\right) \leqslant h_{1} \varphi\left(x_{0}+h_{2} x\right)$ $+\left(h_{2}-h_{1}\right) \varphi\left(x_{11}\right)$ d. h. $h_{2}\left[\varphi\left(x_{0}+h_{1} x\right)-\varphi\left(x_{0}\right)\right] \leqslant h_{1}\left[\varphi\left(x_{0}+h_{2} x\right)-\varphi\left(x_{0}\right)\right]$, also $R\left(x, h_{1}\right) \leqslant R\left(x, h_{2}\right)$. Folglich ist $R(x, h)$ eine nichtabnehmende Funktion von $h$ und, da stets $\varphi\left(x_{0}\right)=\varphi\left(x_{0}+h x-h x\right) \leqslant \varphi\left(x_{0}+h x\right)$ $+h \varphi(-x)$ d. h. $-h \varphi(-x) \leqslant \varphi\left(x_{0}+h x\right)-\varphi\left(x_{0}\right)$, also $-\varphi(-x)$ $\leqslant R(x, h)$, so existiert der endliche $\lim _{h \rightarrow+0} R(x, h)$. Sind $x, y \in E, t$ eine Zahl $>0$, so ist $2 \varphi\left(x_{0}+\frac{h}{2}(x+y)\right)=\varphi\left(x_{0}+h x+x_{0}+h y\right)$ $\leqslant \varphi\left(x_{0}+h x\right)+\varphi\left(x_{0}+h y\right)$, also $2\left[\varphi\left(x_{0}+\frac{h}{2}(x+y)\right)-\varphi\left(x_{0}\right)\right]$ $\leqslant\left[\varphi\left(x_{0}+h x\right)-\varphi\left(x_{0}\right)\right]+\left[\varphi\left(x_{0}+h y\right)-\varphi\left(x_{0}\right)\right]$, woraus $R\left(x+y, \frac{h}{2}\right)$ $\leqslant R(x, h)+R(y, h)$ folgt; sind $x \in E$ und $h, t$ Zahlen $>0$, so ist $R(t x, h)=t R(x, t h)$; ist $x \in E, h$ eine Zahl $>0$, so ist $\varphi\left(x_{0}+h x\right)$ $\leqslant \varphi\left(x_{0}\right)+h \varphi(x)$, also $R(x, h) \leqslant \varphi(x)$. Aus den oben bewiesenen Relationen folgt sofort, daß die Bedingungen $\overline{1^{\circ}}-\overline{3^{\circ}}$ erfüllt sind.

7. Wir nehmen an, daß die Bedingungen von 6 erfüllt sind. Ist $F(x)$ ein in $E$ additives und homogenes Funktional derart, daß $F\left(x_{0}\right)=\varphi\left(x_{0}\right), F(x) \leqslant \varphi(x)$ für $x \in E$, so ist stets $-\Phi(-x) \leqslant F(x)$ $\leqslant \Phi(x)$; ist $x^{*} \in E$ und a eine Zahl, für die $-\Phi\left(-x^{*}\right) \leqslant a \leqslant \Phi\left(x^{*}\right)$, so gibt es in $E$ ein additives und homogenes Funktional $F(x)$ derart, daß $F\left(x_{0}\right)=p\left(x_{0}\right), F\left(x^{*}\right)=a$, sowie $F(x) \leqslant \varphi(x)$ für $x \in E$.Sei $F(x)$ ein in $E$ additives und homogenes Funktional, wobei bemerkt.

7) Dies warde von Herrn Ascoli in der unter ${ }^{1)}$ zitierten Arbeit, p. 53-55, 
$F\left(x_{0}\right)=\varphi\left(x_{0}\right)$ und $F(x) \leqslant \varphi(x)$ für $x \in E$. Ist $x \in E, h$ eine Zahl $>0$, so ist $\varphi\left(x_{0}\right)+h F(x)=F\left(x_{0}+h x\right) \leqslant \varphi\left(x_{0}+h x\right)$, also $F(x)$ $\leqslant \frac{1}{h}\left[\varphi\left(x_{0}+h x\right)-\varphi\left(x_{0}\right)\right]$, was $F(x) \leqslant \Phi(x)$ impliziert; daraus folgt auch $F(x)=-F(-x) \geqslant-\phi(-x)$. Sei jetzt $x^{* *} \in E$ und $a$ eine den Ungleichungen $-\Phi\left(-x^{*}\right) \leqslant \alpha \leqslant \Phi\left(x^{*}\right)$ genügende Zahl. Sei weiter $R$ die lineare aus den Elementen $x=t x_{0}+s x^{*}$, wo $t, s$ Zahlen sind, gebildete Menge und setzen wir $f(x)=\operatorname{tr}\left(x_{0}\right)+s a$. Leicht folgt, daß diese Definition eindeutig und das auf diese Weise in $R$ erklärte Funktional additiv und homogen ist; ferner ist $f(x) \leqslant \Phi(x)$ für $x \in R$. In der Tat sei $x=t x_{0}+s x^{*}$, wo $t, s$ Zahlen sind; für positive Zahlen $h$ ist $\frac{1}{h}\left[p\left(x_{0}+h x\right) \cdots p\left(x_{(1)}\right)\right]$ $=t w\left(x_{0}\right)+\frac{1+t h}{h}\left[\rho\left(x_{0}+\frac{h}{1+t} s x^{*}\right)-i\left(x_{0}\right)\right]$, woraus die Gleich. heit $\Phi(x)=t \varphi\left(x_{0}\right)+\Phi\left(s x^{*}\right)$ folgt; da weiter $f(x)=t \varphi(t)\left(x_{0}\right)+s a$, so ist tatsächlich $f(x) \leqslant \Phi(x)$, weil in Anbetracht der Relation $-\Phi\left(-x^{*}\right) \leqslant a \leqslant \Phi\left(x^{*}\right)$ offenbar $s a \leqslant \Phi\left(s x^{*}\right)$ gilt. Auf 6 bezugnehmend folgern wir jetzt, daß es in $E$ ein additives und homogenes Funktional $F(x)$ gibt derart, daß stets $F(x) \leqslant \Phi(x)$ und außerdem $F(x)=f(x)$ für $\left.x \in R^{6}\right)$; weiter ist, wieder nach 6 , $F(x) \leqslant \varphi(x)$ für $x \in E$ und dabei ersichtlich $F\left(x_{0}\right)=c\left(x_{0}\right), F\left(x^{*}\right)=\alpha$.

Sei nun $K$ ein konvexer, 0 im Inneren enthaltender Körper und $x_{0}$ ein Randpunkt von $K$; setzen wir ferner $L(x)$ $\lim _{h \rightarrow+0} \frac{1}{h}\left[K\left(x_{0}+h x\right)-K\left(x_{0}\right)\right]$ für $x \in E$. Aus 2, 5 und 7 folgt sofort:

8. Stellt die Gleichung $F(x)-1=0$, wo $F(x)$ ein lineares Funktional ist, eine durch $x_{0}$ gehende Stützhyperebene von $K d a r$, so ist stets $-L(-x) \leqslant F(x) \leqslant L(x)$; ist $x^{*} \in E$ und a eine Zahl. mit $-L\left(-x^{*}\right) \leqslant a \leqslant L\left(x^{*}\right)$, so gibt es ein lineares Funktional $F(x)$ derart, daß $F\left(x^{*}\right)=a$ und dabei die Gleichung $F(x)-1=0$ eine durch $x_{0}$ gehende Stützhyperebene von $K$ bestimmt $\left.{ }^{8}\right)$.

9. Sei $\varphi(x)$ ein im separablen Raume $E$ vom. Typus $(B)$ erklärtes Funktional derart, daß für $x, y \in E$ und Zahlen $t>0$ : $1^{\circ} \varphi(x+y) \leqslant \varphi(x)+\varphi(y) ; 2^{\circ} \varphi(t x)=t \varphi(x) ; 3^{\circ} \varphi(x) \leqslant M|x|$ sei (M eine Konstante). Dann bildet die Menge $Z$ der Punkte $x_{0} \in E$,

8) Die erste Hälfte dieser Bemerkung wurde von Herrn Ascoli in der untẹr $\left.{ }^{1}\right)$ zitierten Arbeit, p. 55-56, bewiesen. in denen $\lim _{h \rightarrow 0} \frac{1}{h}\left[\varphi\left(x_{0}+h x\right)-\varphi\left(x_{u}\right)\right]$ für $x \in E$ existiert, eine dichte $G_{\delta}$ - Menge; $E-Z$ ist also von erster Kategorie.-Sei die abzählbare Menge der Punkte $x_{1}, x_{2}, \ldots$ dicht und $Z_{n}$ die Menge der $x_{0} \in E$ für die $\lim _{h \rightarrow 0} \frac{1}{h}\left[\varphi\left(x_{0}+h x_{n}\right)-\varphi\left(x_{0}\right)\right]$ existiert $(n=1,2, \ldots)$. Setzt man, für ein gegebenes $x_{0} \in E, \Phi(x)=\lim _{h \rightarrow+0} \frac{1}{h}\left[\varphi\left(x_{0}+h x\right)-\varphi\left(x_{0}\right)\right]$, so ist nach 6 stets $\phi(x) \leqslant \varphi(x)$ und somit $\Phi(x) \leqslant M|x|$, woraus die Stetigkeit des Funktionals $\Phi(x)$ folgt; da ersichtlich aber, damitfür ein gegebenes $x \in E \lim _{h \rightarrow 0} \frac{1}{h}\left[\varphi\left(x_{0}+h x\right)-\varphi\left(x_{0}\right)\right]$ existiere, notwendig und hinreichend ist, daß $-\Phi(-x)=\Phi(x)$ sei, so ist $x_{0} \in Z$ dann und nur dann, wenn stets $-\Phi\left(-x_{n}\right)=\Phi\left(x_{n}\right)$, d. h. $x_{0} \in Z_{n}$. Somit gilt $Z=Z_{1} Z_{2} \ldots$, und es genügt zu zeigen, daß jede der Mengen $Z_{n}$ eine $F_{r}$-Menge von erster Kategorie ist. Bei natürlichen $n, p, q$ sei $Z_{n p q}$ die Menge der $x_{0} \in E$, für die $\frac{1}{h}\left[\varphi\left(x_{0}+h x_{n}\right)-\varphi\left(x_{0}\right)\right]-\frac{1}{-h}\left[p\left(x_{0}-h x_{n}\right)-\varphi\left(x_{0}\right)\right] \geqslant \frac{1}{p}$ bei $0<h \leqslant \frac{1}{q}$; da das Funktional $\varphi(x)$ stetig ist, so sind die Mengen $Z_{n p q}$ abgeschlossen. Wegen 6 ist aber offenbar $E-Z_{n}=\sum_{p, q=1}^{\infty} Z_{n p q}$, daher bildet $E-Z_{n}$ tatsächlich eine $F_{\sigma}$-Menge. Wäre eine von ihnen, etwa $E-Z_{m}$, von zweiter Kategorie, so würde sie eine Kugel enthalten: es gibt dann ein $x^{*} \in E$ und eine Zahl $r>0$ derart, $\mathrm{da} B$ die Ungleichung $\left|x-x^{*}\right| \leqslant r$ die Relation $x \in E-Z_{m}$ impliziert. Setzt man $a(t)=\varphi\left(x^{*}+t x_{m}\right)$ für jede Zahl $t$, so folgt nach 6 , daß die Funktion $a(t)$ überall enie linksseitige und rechtsseitige Ableitung und also mit Ausnahme einer höchstens abzählbaren Menge von $t$-Werten eine Ableitung besitzt ${ }^{9}$ ). Sei $t_{0}$ eine Zahl, so daß $\left|t_{0}\right| \leqslant \frac{r}{\left|x_{m}\right|}$ und dabei $a^{\prime}\left(t_{0}\right)$ existiert; $x_{0}=x^{*}+t_{0} x_{m}$ gesetzt, ist ersichtlich $x_{0} \in Z_{m}$ und ferner $\left|x_{0}-x^{*}\right| \leqslant r$, entgegen dem Vorangehenden.

Ist $K$ ein konvexer Körper und $x_{\theta}$ ein Randpunkt von $K$, so nennen wir die Hyperebene $H$ eine Tangentialhyperebene von

") S. z. B.: W. Sierpiński, Sur l'ensemble des points angulaires d'une courbe $y=f(x)$, Bull. Acad. Sc. Cracovie, 1912, p. 850-855. 
$K$ (wie auch von seinem Rande $F$ ) im Punkte $x_{0}$, wenn sie die einzige durch $x_{0}$ gehende Stützhyperebene von $K$ ist.

Satz 2. Ist $E$ ein separabler Raum vom Typus $(B)$ und $F$ eine konvexe Hyperfläche in $E$, so bildet die Menge $W$ der Punkte $x_{0} \in F$, in denen die Tangentialhyperebene von $F$ existiert, eine in $F$ dichte $G_{\delta}$ Menge; $F-W$ ist also in $F$ von erster $K a-$ tegorie.

Beweis. Sei $K$ der konvexe Körper, dessen Rand $F$ bildet; man kann voraussetzen, daß 0 ein innerer Punkt von $K$ ist. Nach 2 und 9 ist die Menge $Z$ der Punkte $x_{0} \in E$, in denen $\lim _{h \rightarrow 0} \frac{1}{h}\left[K\left(x_{0}+h x\right)-K\left(x_{0}\right)\right]$ für $x \in E$ existiert, eine dichte $G_{0}$ Menge. $F Z$ ist daher in $F$ eine $G_{f}$-Menge; daß sie dabei in $F$ dicht ist, folgt sofort aus der einfachen Bemerkung, daß wenn $x_{0} \in Z$ und $t$ eine Zahl $>0$ ist, so ist auch $t x_{0} \in Z$. Es genügt noch zu beachten, daß, wegen $8, W=F Z$, um den Beweis zu Ende zu führen.

Betrachten wir insbesondere den konvexen Körper $K_{r}$, welchen die Kugel $|x| \leqslant r(r$ eine Zahl $>0)$ bildet. Nach dem Vorangehenden sowie nach 8 gibt es in dem Randpunkte $x_{0}$ von $K_{r}$ dann und nur dann eine Tangentialhyperebene von $K_{r}$, wenn $\lim _{h \rightarrow 0} \frac{1}{h}\left[\left|x_{0}+h x\right|-\left|x_{0}\right|\right]$ für $x \in E$ existiert; ist $E$ ein separabler Raum vom Typus $(B)$, so bildet auf Grund des Satzes 2 die Menge der Punkte des Randes $F_{r}$ von $K_{r}$, in denen dieses stattfindet, eine in $F_{r}$ dichte $G_{\delta}$-Menge. Die Voraussetzung, daß der Raum $E$ separabel (sowie auch vollständig) ist, kann nicht entbehrt werden.

Im Raume $(M)$ der meßbaren beschränkten Funktionen existiert in keinem. Punkte $x_{0} \lim _{h \rightarrow 0} \frac{1}{h}\left[\left|x_{0}+h x\right|-\left|x_{0}\right|\right]$ für alle $x \in(M)$. - Ohne Einschränkung kann man voraussetzen, daß $\left|x_{0}\right|=1$; sei $\varepsilon= \pm 1$ so, daß die wesentliche obere Grenze der Funktion $\varepsilon x_{0}(t)$ in $I=\langle 0,1\rangle$ gleich 1 ist. Es gibt in $I$ abzählbar viele punktfremde meßbare Mengen $I_{1}, I_{2}, \ldots$, so daß stets $\left|I_{n}\right|>0$ und $\varepsilon x_{0}(t) \geqslant \frac{n}{n+1}$ für $t \in I_{n} ;$ wir setzen $f_{n}(x)=\frac{\varepsilon}{\left|I_{n}\right|} \int_{I_{n}} x(t) d t$ für $x \in(M)(n=1,2, \ldots)$. Da $f_{n}(x)$ lineare Funktionale mit der Norm 1 sind, so ist die Konvergenzmenge $R^{\prime}$ der Folge $\left\{f_{2 n-1}(x)\right\}$ abgeschlossen und folglich $f^{\prime}(x)=\lim _{n \rightarrow \infty} f_{2 n-1}(x)$ ein lineares Funktional in $R^{\prime}$; analog bildet $f^{\prime \prime}(x)=\lim _{n \rightarrow \infty} f_{2 n}(x)$ in der Konvergenzmenge $R^{\prime \prime}$ der Folge $\left\{f_{2 n}(x)\right\}$ ein lineares Funktional. Weil dabei die Funktionale $f^{\prime}(x), f^{\prime \prime}(x)$ die Norm $=1$ besitzen, so gibt es in $(M)$ lineare Funktionale $F^{\prime}(x), F^{\prime \prime}(x)$ so, $\operatorname{daß} F^{\prime}(x)=f^{\prime}(x)$ für $x \in R^{\prime}, F^{\prime \prime}(x)=f^{\prime \prime}(x)$ für $x \in R^{\prime \prime}$ und $\left\|F^{\prime}\right\|=\left\|F^{\prime \prime}\right\|=1$; wegen $f^{\prime}\left(x_{0}\right)=f^{\prime \prime}\left(x_{0}\right)=1$ ist $F^{\prime}\left(x_{0}\right)=F^{\prime \prime}\left(x_{0}\right)=1$ und endlich offenbar $F^{\prime}(x) \equiv F^{\prime \prime}(x)$. Die Gleichungen $F^{\prime}(x)-1=0, F^{\prime \prime}(x)-1=0$ bestimmen zwei verschiedene durch $x_{0}$ gehende Stützhyperebenen der Kugel $|x| \leqslant 1$.

Man kann zeigen, daß in den Räumen $\left(L^{p}\right)$ der mit der $p$-ten Potenz integrierbaren Funktionen und $\left(l^{p}\right)$ der mit der $p$-ten Potenz konvergenten Reihen $(p>1)$ in jedem Punkte $x_{0} \neq=0$ stets $\lim _{h \rightarrow 0} \frac{1}{h}\left[\left|x_{0}+h x\right|-\left|x_{0}\right|\right]$ existiert, und zwar sogar gleichmäßig inbezug auf $x$ in der Kugel $|x| \leqslant 1$; in diesen Räumen gibt es also in jedem Randpunkte einer beliebigen Kugel $K$ eine Tangentialhyperebene von $K$.

In allen unten angegebenen Beispielen bezeichnet $Z$ die Menge der Punkte $x_{0}$ des Raumes, in denen stets der $\lim _{h \rightarrow 0} \frac{1}{h}\left[\left|x_{0}+h x\right|-\left|x_{0}\right|\right]$ existiert; wir bezeichnen ihn mit $w\left(x_{0}, x\right)$.

1. Der Raum der reellen stetigen in einem metrischen vollständigen und kompakten Raume $Q$ erklärten Funktionen $x(q)$; die Verknüpfungen erklären wir wie gewöhnlich, $|x|=\max _{q \in Q}|x(q)| . x_{0} \in Z$ dann und nur dann, wenn es ein $q_{0}$ gibt, so daß stets $\left|x_{0}\left(q_{0}\right)\right|>\left|x_{0}(q)\right|$ für $q \neq=q_{0} ; \Phi\left(x_{0}, x\right)=x\left(q_{0}\right)$.sgn $x_{0}\left(q_{0}\right)$. Dieser Satz rührt von Herrn S. B a na ch her ${ }^{10}$ ).

2. Der Raum der reellen beschränkten in einer abstrakten Menge $Q$ erklärten Funktionen $x(q)$; die Verknüpfungen erklären wir wie gewöhnlich, $|x|=$ obere Grenze $|x(q)| . \quad x_{0} \in Z$ dann und nur dann, wenn es ein $q_{0}$ gibt, so

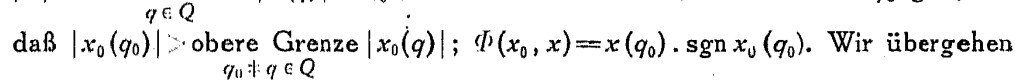
den einfachen Beweis ${ }^{11}$,

3. Der Raum $(L)$ der integrierbaren Funktionen $x \in Z$ dann und nur dann, wenn fast überall $x_{0}(t) \neq 0 ; \phi\left(x_{0} x\right)=\int_{0}^{1} x(t) \operatorname{sgn} x_{0}(t) d t$.

4. Der Raum $(l)$ der absolut konvergenten Reihen.$x_{0} \in Z$ dann und nur dann, wenn stets $\xi_{n}=1=0$, wo $x_{0} \equiv \sum_{n=1}^{\infty} \xi_{n}^{0} ; \Phi\left(x_{0}, x\right) \underset{n=1}{=} \sum_{n}^{\infty} \xi_{n}$. sgn $\xi_{n}^{0}$, wo $x \equiv \sum_{n=1}^{\infty} \xi_{n}$.

Bemerken wir noch, daß in den obigen Beispielen in jedem Punkte $x_{0} \in Z \lim _{h \rightarrow 0} \frac{1}{h}\left[\left|x_{0}+h x\right|-\left|x_{j}\right|\right]$ gleichmäßig inbezug auf $x$ in der Kugel $|x| \leqslant 1$ existiert.

10) S. das unter 9) zitierte Buch, p. 168.

11) Aus dieser Bemerkung folgt leicht: Sind die Räume der reellen, beschränkten, in den abstrakten Mengen $Q^{r}$ bzw. $Q^{\prime \prime}$ erklärten Funktionen isometrisch, so sind die Mengen $Q^{\prime}$ und $Q^{\prime \prime}$ gleichmächtig. 


\section{$\S 2$.}

Sei $E$ ein linearer, normierter Raum. Eine Menge $Z \subset E$ nennen wir schwachabgeschlossen, wenn daraus, daß $x_{n} \in Z$ $(n=1,2, \ldots)$ und die Folge $\left\{x_{n}\right\}$ gegen $x_{0} \in E$ schwach konvergiert, $x_{0} \in E$ folgt; die Folge $\left\{x_{n}\right\}$ konvergiert schwach gegen $x_{0}$, wenn für jedes in $E$ lineare Funktional $F(x)$ die Folge $\left\{F\left(x_{n}\right)\right\}$ gegen $F\left(x_{0}\right)$ konvergiert. Jede schwachabgeschlossene Menge ist offenbar abgeschlossen, nicht aber umgekehrt. Die Kugeln bilden schwachabgeschlossene Mengen. Sei nämlich die Folge $\left\{x_{n}\right\}$ gegen $x_{0}$ schwachkonvergent und stets $\left|x_{n}\right| \leqslant r, r$ eine Zahl $>0$; ist $F(x)$ ein lineares Funktional derart, daf3 $\|F\|=1, F\left(x_{0}\right)=\left|x_{0}\right|$, so ist $F\left(x_{n}\right) \leqslant\left|x_{n}\right|$ d. h. $F\left(x_{n}\right) \leqslant r$, was $F\left(x_{11}\right) \leqslant r$ und also $\left|x_{1}\right|<r$, impliziert. Ebenso leicht beweist man, daf lineare abgeschlossene Mengen und somit auch lineare abgeschlossene Mannigfaltigkeiten schwachabgeschlossen sind. Die erwähnten Sätze sind Spezialfälle des allgemeinen unten zu beweisenden Satzes, laut dessen jede konvexe abgeschlossene Menge schon schwachabgeschlossen ist.

10. Ist W eine konvexe abgeschlossene (beschränkte) Menge und $y_{0} \in E-W$, so gibt es einen konvexen (beschränkten) Körper $K$, so $d a \beta W \in K, y_{0} \in E-K$. - Sei $d$ die Entfernung des Punktes $y_{0}$ von der Menge $W$. Für $x \in W$ bezeichnen wir mit $C_{x}$ die offene Kugel vom Mittelpunkt $x$ und Radius $d / 2 ; V$ sei die Vereinigung der Kugeln $C_{x}$. Die Menge $V$ ist offen und dabei konvex. In der Tat sei $y_{1}, y_{2} \in V$ und $y=t y_{1}+(1-\dot{t}) y_{2}$, wo $t \in\langle 0,1\rangle$; es gibt Punkte $x_{1}, x_{2} \in W$, so daß $y_{1} \in C_{x_{1}}, y_{2} \in C_{x_{2}}$, und da $W$ konvex ist, so ist $x=t x_{1}+(1-t) x_{2}$ ein Element von $W$; aus $|y-x|$ $\leqslant t\left|y_{1}-x_{1}\right|+(1-t)\left|y_{2}-x_{2}\right|<d / 2$ lolgt $y \in C_{x}$ und somit $y \in V$. Die Entfernung des Punktes $y_{0}$ von der Menge $W$ ist offenbar $-d / 2$. Die abgeschlossene Hülle $K$ der Menge $V$ bildet also einen konvexen Körper mit den geforderten Eigenschaften.

Satz 3. Eine konvexe abgeschlossene Menge W ist schwach abgeschlossen.

Be weis. Man kann voraussetzen, daß $0 \in W$. Nehnen wir an, daß $y_{n} \in W(n=1,2, \ldots)$, die Folge $\left\{y_{n}\right\}$ gegen $y_{0}$ schwach konvergiert und dabei $y_{0} \in E-W$; nach 10 gibt es einen konvexen Körper $K$, so daß $W \subset K, y_{0} \in E-K$. Sei a die größte Zahl, für die $a y_{0} \in K$; ersichtlich ist $0<a<1$ und der Punkt $x_{0}=a y_{0}$ gehört dem Rande von $K$ an. Auf Grund des Satzes 1 gibt es eine durch $x_{0}$ gehende Stützhyperebene $H$ von $K$; sei $F(x)-1=0$, wo $F(x)$ ein lineares Funktional ist, die Gleichung von $H$. Wegen $F(0)-1<0$, ist $F(x)-1 \leqslant 0$ für $x \in K$; insbesondere also ist stets $F\left(y_{n}\right) \leqslant 1$, woraus $F\left(y_{0}\right) \leqslant 1$ folgt. Dies widerspricht der Relation $F\left(y_{0}\right)=\frac{1}{a} F\left(x_{0}\right)=\frac{1}{a}>1$.

11. Ist die Folge $\left\{x_{n}\right\}$ gegen $x_{0}$ schwachkonvergent, so gibt es zu jeder Zahl $\varepsilon>0$ Zahlen $c_{n} \geqslant 0(n=1,2, \ldots m)$, so daß $\sum_{n=1}^{m} c_{n}=1$ und $\left|\sum_{n=1}^{m} c_{n} x_{n}-x_{0}\right|<\varepsilon .-V$ bezeichne die Menge der Elemente der Form $\sum_{n=1}^{m} c_{n} x_{n}$, wo $c_{n}(n=1,2, \ldots m)$ Zahlen $\geqslant 0$ mit der Summe 1 sind $(m=1,2, \ldots)$; die Menge $V$ ist ersichtlich konvex, ihre abgeschlossene Hülle $W$ ist also eine konvexe abgeschlossene Menge. Da stets $x_{n} \in V$, somit auch $x_{n} \in W$, und die Folge $\left\{x_{n}\right\}$ gegen $x_{0}$ schwach konvergiert, so ist auf Grund des Satzes $3 \quad x_{0} \in W$.

Ist die Folge $\left\{x_{n}\right\}$ gegen $x_{0}$ schwachkonvergent, so enthält jede konvexe abgeschlossene Menge - insbesondere also jeder konvexe Körper - mit unendlich vielen Gliedern der Folge $\left\{x_{n}\right\}$ auch $x_{0}$; dies folgt aus dem Satze 3. Es gilt auch der folgende

Satz 4. Enthält jeder konvexe (beschränkte) Körper mit unendlich vielen Gliedern der (beschränkten) Folge $\left\{x_{n}\right\}$ auch $x_{0}$, so ist die Folge $\left\{x_{n}\right\}$ gegen $x_{0}$ schwachkonvergent.

Beweis. Wäre die Behauptung nicht richtig, so gäbe es ein lineares Funktional $F(x)$ derart, daß die Folge $\left\{F\left(x_{n}\right)\right\}$ gegen $F\left(x_{0}\right)$ nicht konvergierte. Sei $\left\{k_{n}\right\}$ eine wachsende Indexfolge, so daß die Folge $\left\{F\left(x_{k_{n}}\right)\right\}$ eine von $F\left(x_{0}\right)$ verschiedene Grenze besitzt; wir können annehmen, daß stets $F\left(x_{k_{n}}-x_{0}\right) \geqslant 1$. Sei nun $W$ die abgeschlossene Hülle der aus den Elementen der Form $\sum_{n=1}^{m} c_{n} x_{k_{n}}$, wo $c_{n}(n=1,2, \ldots)$ Zahlen $\geqslant 0$ mit der Summe 1 sind, gebildete Menge; $W$ ist konvex, abgeschlossen (beschränkt) und dabei $x_{0} \in E-W$. Anderenfalls gibt es nämlich Zahlen $c_{n} \geqslant 0(n=1,2, \ldots m)$ mit der Summe 1, so daß $\left|\sum_{n=1}^{m} c_{n} x_{k_{n}}-x_{0}\right|<\frac{1}{\|F\|}$, also $\sum_{n=1}^{m} c_{n} F\left(x_{k_{n}}\right)$ 
$-F\left(x_{0}\right)=F\left(\sum_{n=1}^{m} c_{n} x_{k_{n}}-x_{0}\right) \leqslant\|F\|\left|\sum_{n=1}^{m} c_{n} x_{k_{n}}-x_{0}\right|<1$; das aber ist unmöglich, denn $\sum_{n=1}^{m} c_{n} F\left(x_{k_{n}}\right)-F\left(x_{0}\right)=\sum_{n=1}^{m} c_{n} F\left(x_{k_{n}}-x_{0}\right)$ $\geqslant \sum_{n=1}^{m} c_{n}=1$. Nach 10 gibt es einen konvexen (beschränkten) Körper $K$, so daß $W \subset K, x_{0} \in E-K$; es ist also $x_{k_{n}} \in K(n=1,2, \ldots)$ und $x_{0} \in E-K$, was im Widerspruche zur Voraussetzung steht.

Der Punkt $x_{0}$ heißt Mittelpunkt der Menge $Z$, wenn aus $x \in Z$ stets $2 x_{0}-x \in Z$ folgt. Eine beschränkte Menge kann höchstens einen Mittelpunkt besitzen; ein Mittelpunkt einer konvexen Menge gehört ibr an, und ein Mittelpunkt eines konvexen Körpers $K$ ist sogar ein innerer Punkt von $K$.

12. Ist $W$ einc konvexe, abgeschlossene und beschränkte Menge, $\dot{y}_{0} \in E-W$, so gibt ès einen konvexen beschränkten Körper $K$ mit Mittelpunkt, so das $W \subset K, y_{0} \in E-K$. - Nach 10 gibt es nämlich einen konvexen beschränkten Körper $K^{*}$, so daß $W \subset K^{*}$, $y_{0} \in E-K^{*}$. Sei $x_{0}$ ein innerer Punkt von $K^{*}$ und $a$ eine Zahl, für die $0<a<1, z_{0}=(1-a) x_{0}+a y_{0} \in E-K^{*} ; S$ bezeichne die Menge der Elemente der Form $(1-t) z_{0}+t x$, wo $x \in K^{*}, t$ eine Zahl $\geqslant 0$ ist. Es ist $K^{*} C S$, und da die Menge $S$ konvex sowie abgeschlossen ist, so bildet sie einen konvexen Körper; dabei ist aber $y_{0} \in E-S$. Anderenfalls gibt es ein $x^{*} \in K^{*}$ und eine Zahl $t_{0} \geqslant 0$, so daß $y_{0}=\left(1-t_{0}\right) z_{0}+t_{0} x^{*}$, also $y_{0}=(1-b) x_{0}+b x^{*}$, wo $b=\frac{t_{0}}{1-a\left(1-t_{0}\right)}$; daraus folgt sofort wegen $x_{0}, x^{*} \in K^{*}$ und $0<b<1$ die Relation $y_{0} \in K^{*}$, entgegen dem Vorangehenden. Da $\lim _{n \rightarrow \infty}\left(\frac{2 n}{2 n-1} x_{0}-\frac{1}{2 n-1} x\right)=x_{0}$ gleichmäßig inbezug auf $x$ in $K^{*}$, so gibt es eine natürliche Zahl $m$ derart, daß $\frac{2 m}{2 m-1} x_{0}-\frac{1}{2 m-1} x \in K^{*}$ für $x \in K^{*}$. Sei $T$ die Menge der Elemente der Form $2\left[(1-m) z_{0}+m x_{0}\right]-x$, wo $x \in S ; T$ ist ersichtlich ein konvexer Körper. Es ist dabei $K^{*} C T$ d. h. $2\left[(1-m) z_{0}+m x_{0}\right]-x \in S$ für $x \in K^{*}$, da stets $2\left[(1-m) z_{0}+m x_{0}\right]-x=[1-(2 m-1)] z_{0}+$ $(2 m-1)\left(\frac{2 m}{2 m-1} x_{0}-\frac{1}{2 m-1} x\right)$. Setzen wir $K=S T$; dann ist $K^{*} C K$, also auch $W \subset K ; K$ bildet einen konvexen Körper mit $(1-m) z_{0}+m x_{0}$ als Mittelpunkt und $y_{0} \in E-K$. Bleibt noch zu zeigen, daß $K$ beschränkt ist. Nehmen wir an, daß $y_{n} \in K(n=1,2, \ldots), \lim \left|y_{n}\right|=+\infty$; es ist stets $y_{n}=\left(1-t_{n}^{\prime}\right) z_{0}+t_{n}^{\prime} x_{n}^{\prime}=2\left[(1-m) z_{0}+m x_{0}\right]-\left[\left(1-t_{n}^{\prime \prime}\right) z_{0}\right.$ $\left.+t_{n}^{\prime \prime} x_{n}^{\prime \prime}\right]$, wo $x_{n}^{\prime}, x_{n}^{\prime \prime} \in K^{*}$ und $t_{n}^{\prime \prime}, t_{n}^{\prime \prime}$ Zahlen $\geqslant 0$ sind. Daraus folgt sofort $\frac{t_{n}^{\prime}}{t_{n}^{\prime}+t_{n}^{\prime \prime}} x_{n}^{\prime}+\frac{t_{n}^{\prime \prime}}{t_{n}^{\prime}+t_{n}^{\prime \prime}} x_{n}^{\prime \prime}=z_{0}+\frac{1}{t_{n}^{\prime}+t_{n}^{\prime \prime}} y_{0}$ für $t_{n}^{\prime}+t_{n}^{\prime \prime} \neq 0$, und da $\frac{t_{n}^{\prime}}{t_{n}^{\prime}+t_{n}^{\prime \prime}} x_{n}^{\prime}+\frac{t_{n}^{\prime \prime}}{t_{n}^{\prime}+t_{n}^{\prime \prime}} x_{n}^{\prime \prime} \in K^{*}$ und $\lim _{n \rightarrow \infty}\left(t_{n}^{\prime}+t_{n}^{\prime \prime}\right)=+\infty$, so ist $z_{0} \in K^{*}$, entgegen der Annahme.

Aus dem Satze 4 und 12 folgt

13. Enthält jeder konvexe beschränkte Körpèr mit Mittelpunkt mit unendlich vielen Gliedern der beschränkten Folge $\left\{x_{n}\right\}$ auch $x_{0}$, so ist die Folge $\left\{x_{n}\right\}$ gegen $x_{0}$ schwachkonvergent.

Bekanntlich kann man im Falle eines euklidischen Raumes die Bemerkung 12 dahin verschärfen, daß es eine Kugel $K$ gibt derart, daß $\left.W \subset K, y_{1} \in K^{12}\right)$. In allgemeinen linearen normierten Räumen findet das offenbar nicht statt ${ }^{13}$ ); es sind spezielle Voraussetzungen über den Raum notwendig. Macht man sie, so ist nach dem Satze 4 die beschränkte Folge $\left\{x_{n}\right\}$ gegen $x_{0}$ schwachkonvergent, wenn jede Kugel mit unendlich vielen Gliedern der Folge $\left\{x_{n}\right\}$ auch $x_{0}$ enthält. Wir befassen uns damit an anderer Stelle und zeigen, daß der oben ausgesprochene Satz im besonderen für die Räume $\left(L^{p}\right),\left(l^{p}\right)(p>1)$ gilt.

Sei $K$ ein konvexer beschränkter Körper mit 0 als Mittelpunkt. Aus 1, 4 und der Tatsache, daß stets $K(-x)=K(x)$, folgt sofort, daß das Funktional $K(x)$ eine Norm in $E$ bildet d. h. daß die folgenden Bedingungen erfült sind $\left(x, y \in E, \quad t\right.$ eine Zahl ist): $1^{\circ} K(x)>0$ für $x \neq 0, K(0)=0 ; 2^{\circ} K(x+y)$ $\leqslant K(x)+K(y) ; 3^{\circ} K(t x)=|t| K(x)$ - und dabei ist die Norm $K(x)$ mit der Norm $|x|$ äquivalent, d. h. für $x_{n} \in E(n=1,2, \ldots)$ ist $\lim _{n \rightarrow \infty} K\left(x_{n}\right)=0$ dann und nur dann wenn $\lim _{n \rightarrow \infty}\left|x_{n}\right|=0$. Bei der Norm $K(x)$ wird $E$ zu einem linearen normierten Raume $E(K) ; E(K)$ ist mit dem ursprünglichen Raume $E$ isomorph. Seien $K^{\prime}, K^{\prime \prime}$ zwei konvexe beschränkte Körper mit 0 als Mittelpunkt; man kann zeigen, daß, damit die Räume $E\left(K^{\prime}\right), E\left(K^{\prime \prime}\right)$, isometrisch seien, notwendig

12) Vgl. z. B.: S. Straszewicz, Przyczynek do teorji mnogości wypukkych, Prace mat.-fiz. 27 (1916) p. 1-10, insb. p. 10.

111) Vgl.: S. Mazur, Über die kleinste konvexe Menge, die eine gegebene kompakte Menge enthält, Stud. Math. 2 (1930) p. 7-9, insb. p. 9. 
und hinreichend ist, da $\beta$ die Mengen $K^{\prime}, K^{\prime \prime}$ affin seien $\left.{ }^{14}\right)$. Dabei nennen wir zwei Mengen affin, wenn es eine isomorphe Abbildung des Raumes $E$ auf sich selbst gibt, die eine von ihnen in die andere überführt. Umgrekehrt sei nun ein in $E$ erklärtes Funktional $F(x)$ eine mit $|x|$ äquivalente Norm; auf Grund von 3,4 und dessen, daß stets $K(-x)=K(x)$, ist die durch $K(x) \leqslant 1$ bestimmte Menge $K$ ein konvexer beschränkter Körper mit 0 als Mittelpunkt; $K(x)$ ist das Minkowskische Funktional von $K$. Endlich bezeichne $E^{*}$ einen linearen normierten mit $E$ isomorphen Raum und $U(x)$ eine isomorphe Abbildung von $E$ auf $E^{4}$; es ist klar, daß die durch $|U(x)| \leqslant 1$ bestimmte Menge einen konvexen beschränkten Körper $K$ mit 0 als Mittelpunkt bildet; ferner ist $E^{\text {* }}$ mit $E(K)$ isometrisch. Zusammenfassend sieht man, daß wenn $M$ die Klasse aller Räume $E(K)$ ( $K$ ein konvexer beschränkter Körper mit 0 als Mittelpunkt) bezeichnet. so ist jeder Raum der Klasse $M$ mit $E$ isomorph; jeder lineare normierte mit $E$ isomorphe Raum ist mit einem gewissen Raume der Klasse $M$ isometrisch. Wenn $E$ speziell der $n$-dimensionale euklidische Raum ist, so besteht die Klasse $M$ aus allen $n$-dimensionalen Minkowskischen Räumen; da alle $n$-dimensionalen, linearen, normierten Räume isomorph sind, so ist nach dem Vorangehenden jeder $n$-dimensionale lineare normierte Raum mit einem $n$-dimensionalen Min* kowskischen Raume isometrisch.

(Reçu par la Rédaction le 6. 6. 1933).

14) Wenn $U(x)$ eine isometrische Abbildung des linearen normierten Raumes $X$ auf einen ebensolchen Raum $Y$ bildet und $U(0)=0$, so ist $U(x)$ linear: S. Mazur et S. Ulam, Sur les transformations isométriques d'espaces vectoriels normés, C. R. 194 (1932) p. 946-948.

\section{Eine Bemerkung zum starken Gesetz der großen Zahlen}

von

Z. W. BIRNBAUM und J. SCHREIER (Lwów).

Die von Herrn R. v. Mises formulierte „Regellosigkeitsforderung", welche von ihm auch als das "Prinzip vom ausgeschlossenen Spielsystem" bezeichnet wird, hat zu einer lebhaften Diskussion und zahlreichen Einzeluntersuchungen Anlaß gegeben. Es dürfte deshalb vielleicht nicht ohne Interesse sein, das Verhalten einer Folge von Wiederholungen desselben Spieles gegenüber einer, auf Grund eines im voraus gegebenen Spielsystems vorgenommenen Stellenauswahl zu untersuchen. In der Folge soll gezeigt werden, daß das "starke Gesetz der großen Zahlen“ auch in diesem Falle gilt.

Wir betrachten ein gerechtes Spiel, bei welchem nur zwei Ergebnisse $e$ bzw. $\bar{e}$ mit den Wahrscheinlichkeiten $p$ bzw. $1-p=q$ möglich sind (der Fall eines Spieles mit mehr als zwei möglichen Ergebnissen kann ganz analog behandelt, oder auch auf den Fall eines zweiwertigen Spieles zurückgeführt werden). Einem solchen Spiel entspricht eine auf den Erwartungswert Null normierte Zufallsvariable, welche den Wert 1/p mit der Wahrscheinlichkeit $p$ und den Wert $-1 / q$ mit der Wahrscheinlichkeit $q$ annimmt. Dem abzählbar oft wiederholten Spiel kann bekanntlich folgendes arithmetische Modell zugeordnet werden:

Das Intervall $\langle 0,1\rangle$ wird in zwei Teile, $I_{0}$ von der Länge $\left|I_{0}\right|=p$ und $I_{1}$ von der Länge $\left|I_{1}\right|=q$ zerlegt. $I_{0}$ teilt man in $I_{00}$ mit der Länge $\left|I_{00}\right|=p^{2}$ und $I_{01}$ mit der Länge $\left|I_{01}\right|=p q$, $I_{1}$ wird in $I_{10}$ mit der Länge $\left|I_{10}\right|=q p$ und $I_{11}$ mit der Länge $\left|I_{11}\right|=q^{2}$ zerlegt. Eine Fortsetzung dieses Verfahrens liefert die Intervalle $I_{v_{1} v_{2}} \cdots_{n_{n}}$ für beliebige natürliche $n$ und alle möglichen 